\title{
Analytical expression of top surface charge sensitivity in fully depleted semiconductor on insulator MOS transistor
}

\section{Expression analytique de la sensibilité de la charge de surface avant pour les transistors MOS semiconducteur sur isolant complètement déplétés}

\author{
G. Ghibaudo ${ }^{1}$, G. Pananakakis ${ }^{1}$ \\ ${ }^{1}$ IMEP-LAHC, Univ. Grenoble Alpes, Minatec/INPG, 3 Parvis Louis Neel, 38016 Grenoble, France
}

\begin{abstract}
An analytical expression of free top surface charge sensitivity in FDSOI MOS structure has been established for weak inversion region and validated by TCAD numerical simulation. The influence of various FDSOI stack parameters has been analyzed. The impact of the interface trap density has been particularly emphasized, leading to a strong undesired degradation of sensitivity. This indicates that top surface passivation is a key issue for efficient charge sensing. These expressions of top surface charge sensitivity and associated threshold voltage shift should be very useful for sensor design and electrical characterization purpose.

KEYWORDS. charge sensitivity, FDSOI, threshold voltage, analytical expression.
\end{abstract}

\section{Introduction}

Field effect transistor (FET) based sensors are very attractive for their advantages in terms of miniaturization, integration, label-free detection and high sensitivity [BER03, STE07]. In this context, Ion Sensitive Field Effect Transistors (ISFET) have found applications as $\mathrm{pH}$ sensors. To improve the sensitivity of single gate ISFET, dual or double gate MOS devices with top and bottom gate electrodes have been introduced [IBA05, PAR14]. More recently, industrial grade FDSOI technology was employed to fabricate high sensitivity $\mathrm{pH}$ sensors, exploiting the huge front to back gate coupling in such fully depleted thin-film FET structure [MON16, RAH17]. In these FET sensors, the sensitivity is maximized when the transistor is operated in weak inversion i.e. subthreshold region, where front to back gate electrostatic coupling is not screened out by the channel formation.

Therefore, in this work, we aim at developing an analytical expression for the top surface charge sensitivity of FDSOI MOS transistors operated in weak inversion. This analytical expression could find several applications in FET sensor architecture design and electrical characterization.

\section{Analytical modeling of surface charge sensitivity in FDSOI structure}

In a recent paper [GHI18], we have proposed analytical expressions for the subthreshold swing in FDSOI structure. To this aim, we have considered a 1D FDSOI structure (see Fig. 1) consisting of a fully depleted silicon film of thickness $t_{\mathrm{si}}$ (associated capacitance $\mathrm{C}_{\mathrm{si}}$ ) sandwiched by a top gate oxide of equivalent thickness $\mathrm{t}_{\mathrm{ox} 2}$ (associated capacitance $\mathrm{C}_{\mathrm{ox} 2}$ ) and a bottom oxide of equivalent thickness $\mathrm{t}_{\mathrm{ox} 1}$ (associated capacitance $\mathrm{C}_{\mathrm{ox} 1}$ ). The bottom (resp. top) silicon channel interface features an interface trap density $\mathrm{N}_{\mathrm{it} 1}$ (resp. $\mathrm{N}_{\mathrm{it} 2}$ ) with associated capacitance $\mathrm{C}_{\mathrm{it1}}=\mathrm{q} \cdot \mathrm{N}_{\mathrm{it} 1}\left(\operatorname{resp} . \mathrm{C}_{\mathrm{it} 2}=\mathrm{q} \cdot \mathrm{N}_{\mathrm{it} 2}\right)$. Gate voltage $\mathrm{V}_{\mathrm{g} 1}$ (resp. $\mathrm{V}_{\mathrm{g} 2}$ ) is directly applied to the bottom gate oxide (resp. top gate oxide). For free top surface, $\mathrm{V}_{\mathrm{g} 2}$ is no longer applied on the top gate but is related to the top surface electric field controlled by the external charge $\mathrm{Q}_{\text {ext }}$ (see Eq. (1c) below). For simplicity, we assume undoped silicon film, which will have no impact on the subsequent charge sensitivity derivation. 


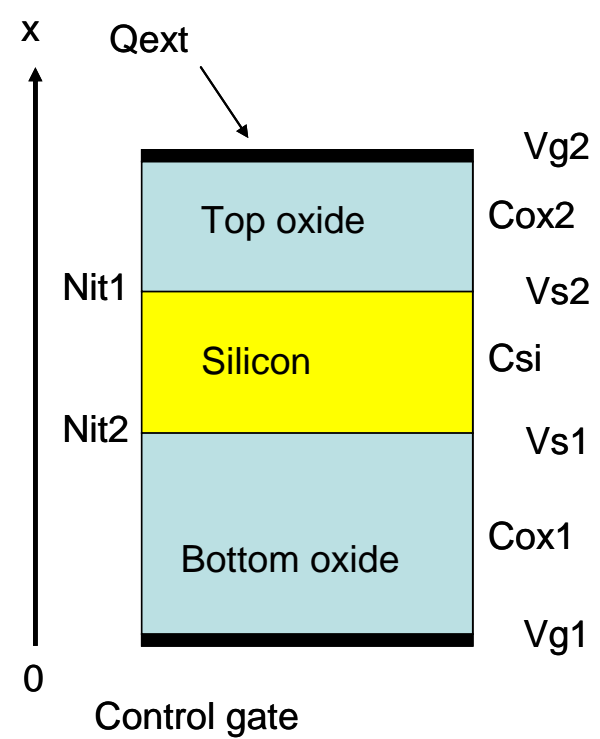

Figure 1. Schematic of $1 D$ FDSOI structure under consideration for analytical calculation of top surface charge sensitivity.

Now, considering as in previous works [BAL90, WOU90, MAZ91, GHI18], that the free carrier charge in the channel can be neglected, which is of course verified in subthreshold region, and by turn, that the potential across the structure varies linearly with space, it is easy to show from Gauss's law applied at top and bottom interfaces, that the front and back surface potentials are related by:

$$
\begin{aligned}
& V_{s 1}=\frac{Q_{i t 1}}{C_{s i}+C_{o x 1}}+\frac{C_{s i}}{C_{s i}+C_{o x 1}} \cdot V_{s 2}+\frac{C_{o x 1}}{C_{s i}+C_{o x 1}} \cdot V_{g 1} \\
& V_{s 2}=\frac{Q_{i t 2}}{C_{s i}+C_{o x 2}}+\frac{C_{s i}}{C_{s i}+C_{o x 2}} \cdot V_{s 1}+\frac{C_{o x 2}}{C_{s i}+C_{o x 2}} \cdot V_{g 2} \\
& V_{g 2}=-\frac{Q_{e x t}}{C_{o x 2}}+V_{s 2}
\end{aligned}
$$

where $\mathrm{Q}_{\mathrm{it} 1}=-\mathrm{q} \cdot \mathrm{N}_{\mathrm{it} 1} \cdot \mathrm{V}_{\mathrm{s} 1}$ and $\mathrm{Q}_{\mathrm{it} 2}=-\mathrm{q} \cdot \mathrm{N}_{\mathrm{it} 2} \cdot \mathrm{V}_{\mathrm{s} 2}$ are the interface trap charges, $\mathrm{q}$ being the absolute electron charge.

After solving the system of Eqs (1) for the unknown $V_{s 1}$ and $V_{s 2}$ one obtains:

$$
\begin{aligned}
& V_{s 1}=\frac{C_{s i} \cdot C_{o x 1} \cdot V_{g 1}+C_{o x 1} \cdot C_{i t 2} \cdot V_{g 1}-C_{s i} \cdot Q_{e x t}}{C_{s i} \cdot C_{o x 1}+C_{s i} \cdot C_{i t 1}+C_{s i} \cdot C_{i t 2}+C_{o x 1} \cdot C_{i t 2}+C_{i t 1} \cdot C_{i t 2}} \\
& V_{s 2}=\frac{C_{s i} \cdot C_{o x 1} \cdot V_{g 1}-C_{s i} \cdot Q_{e x t}-C_{o x 1} \cdot Q_{e x t}-C_{i t 1} \cdot Q_{e x t}}{C_{s i} \cdot C_{o x 1}+C_{s i} \cdot C_{i t 1}+C_{s i} \cdot C_{i t 2}+C_{o x 1} \cdot C_{i t 2}+C_{i t 1} \cdot C_{i t 2}} .
\end{aligned}
$$

Note that, in Eq. (2), we have overlooked the flat band voltage $V_{\mathrm{fb} 1}$. Otherwise, we should replace $\mathrm{V}_{\mathrm{g} 1}$ by $\left(\mathrm{V}_{\mathrm{g} 1}-\mathrm{V}_{\mathrm{fb} 1}\right)$ to account for it in Eq. (2).

Following [BAL90, GHI18], the free carrier inversion charge Qi can then be obtained by integration over silicon channel as:

$$
Q_{i}=\int_{0}^{t s i} q \cdot n(x) \cdot d x=q \cdot n_{i} \cdot \frac{k T \cdot t_{s i}}{V_{s 1}-V_{s 2}} \cdot\left[\exp \left(\frac{q V_{s 1}}{k T}\right)-\exp \left(\frac{q V_{s 2}}{k T}\right)\right]
$$

where $n_{i}$ is the intrinsic carrier density. 
The top surface charge sensitivity, Sens $=\delta \log \left(\mathrm{Q}_{\mathrm{i}}\right) / \delta \mathrm{Q}_{\mathrm{ext}}$, can be computed with no approximation using Eqs (2) and (3), providing an exact calculation for the sensitivity. But, this set of equations does not constitute a close form analytical expression for the sensitivity. Therefore, we go one step further by rewriting Eq. (3) in the form:

$$
\ln \left(Q_{i}\right)=\ln \left(q \cdot n_{i} \cdot k T \cdot t_{s i}\right)+\frac{q V_{s 1}}{k T}+\ln \left[\frac{1-\exp \left(-\frac{q \Delta V_{s 12}}{k T}\right)}{\Delta V_{s 12}}\right]
$$

where $\Delta \mathrm{V}_{\mathrm{s} 12}=\mathrm{V}_{\mathrm{s} 1}-\mathrm{V}_{\mathrm{s} 2}$. Moreover, noting that $\mathrm{V}_{\mathrm{s} 1}$ and $\mathrm{V}_{\mathrm{s} 2}$ are very close in weak inversion, we make a first order expansion of Eq. (4) in $\Delta \mathrm{V}_{\mathrm{s} 12}$, providing,

$$
\ln \left(Q_{i}\right) \approx \ln \left(q \cdot n_{i} \cdot k T \cdot t_{s i}\right)+\frac{q V_{s 1}}{k T}-\frac{q \Delta V_{s 12}}{2 k T}=\ln \left(q \cdot n_{i} \cdot k T \cdot t_{s i}\right)+\frac{q V_{s 1}}{2 k T}+\frac{q V_{s 2}}{2 k T} .
$$

Then, we can evaluate the sensitivity from,

$$
\text { Sens }=\frac{\delta \ln \left(Q_{i}\right)}{\delta Q_{\text {ext }}}=\frac{q}{2 . k T}\left(\frac{\delta V_{s 1}}{\delta Q_{\text {ext }}}+\frac{\delta V_{s 2}}{\delta Q_{\text {ext }}}\right)
$$

which becomes using Eq. (2),

$$
\text { Sens }=\frac{q}{2 . k T} \frac{2 \cdot C_{s i}+C_{o x 1}+C_{i t 1}}{C_{s i} \cdot C_{o x 1}+C_{s i} \cdot C_{i t 1}+C_{s i} \cdot C_{i t 2}+C_{o x 1} \cdot C_{i t 2}+C_{i t 1} \cdot C_{i t 2}} .
$$

As will be shown below from numerical simulation, Eq. (7) does provide an improved analytical expression for the top surface charge sensitivity in bottom gate operated FDSOI structures.

Finally, it is worth evaluating the threshold voltage shift $\Delta \mathrm{V}_{\text {th }}$ induced by the top surface external charge. To this end, we use the subthreshold swing (SW) expression found for FDSOI structure in [GHI18] and recalled in Eq. (8),

$$
S W=\frac{\delta V_{g 1}}{\delta \ln \left(Q_{i}\right)}=\frac{k T}{q}\left(1+\frac{C_{i t 1}}{C_{o x 1}}+\frac{\left(2 C_{s i}+C_{o x 1}\right) \cdot C_{i t 2}+C_{i t 1} \cdot C_{i t 2}}{C_{o x 1} \cdot\left(2 C_{s i}+C_{i t 2}\right)}\right)
$$

In order to derive the threshold voltage shift as $\Delta \mathrm{V}_{\mathrm{th}}=$ Sens.SW. $\Delta \mathrm{Q}_{\mathrm{ext}}$, which leads to the expression after combining Eqs (7) and (8):

$$
\Delta V_{t h}=\frac{2 C_{s i}+C_{o x 1}+C_{i t 1}}{C_{o x} \cdot\left(2 C_{s i}+C_{i t 2}\right)} \cdot \Delta Q_{e x t}
$$

In absence of interface traps i.e. $\mathrm{C}_{\mathrm{it} 1}=\mathrm{C}_{\mathrm{it} 2}=0$, this expression reduces to,

$$
\Delta V_{t h}=\frac{1}{C_{o x}}\left(1+\frac{C_{o x}}{2 C_{s i}}\right) \cdot \Delta Q_{e x t}
$$

Note that $\Delta \mathrm{V}_{\mathrm{th}}$ is not simply equal to $\Delta \mathrm{Q}_{\mathrm{ext}} / \mathrm{C}_{\mathrm{ox}}$ as it is often mentioned in the literature.

Moreover, looking at Eqs (7) and (10), it should be noted that the top surface charge sensitivity Sens and the associated threshold voltage shift $\Delta \mathrm{V}_{\text {th }}$ are independent of the top oxide thickness $\mathrm{t}_{\mathrm{ox} 2}$. Actually, this is due to the fact that, in the case of a free surface configuration, the top surface is field (or charge) controlled and no longer voltage controlled, fully justifying this feature. 


\section{Results and discussion}

In order to check the validity of the new analytical expression of Eq. (7) for the sensitivity Sens, we have analyzed the influence of the FDSOI stack parameters and trap density. A nominal FDSOI structure with $\mathrm{t}_{\mathrm{si}}=10 \mathrm{~nm}, \mathrm{t}_{\mathrm{ox} 1}=10 \mathrm{~nm}$ and $\mathrm{t}_{\mathrm{ox} 2}=2 \mathrm{~nm}$ was considered unless specified. The analytical results were compared to exact simulations obtained by TCAD numerical resolution of the Poisson equation under classical statistics.

\subsection{Influence of back gate oxide thickness}

Typical variations of sensitivity Sens with back gate oxide thickness $t_{\mathrm{ox} 1}$ are illustrated in Fig. 2 as obtained from exact calculations (red solid lines) and the analytical expression of Eq. (7) for two interface trap densities with $\mathrm{N}_{\mathrm{it} 1}=\mathrm{N}_{\mathrm{it} 2}$ in the case of bottom gate operated FDSOI structure $\left(\mathrm{t}_{\mathrm{si}}=10 \mathrm{~nm}\right.$, $\mathrm{V}_{\mathrm{g} 1}=0.01 \mathrm{~V}$ ). Note the very good agreement obtained with the analytical formula of Eq. (7), especially for large interface trap density. It is worth noting that, for zero interface trap density, the sensitivity varies linearly with $\mathrm{t}_{\mathrm{ox} 1}$, whereas it saturates rapidly with $\mathrm{t}_{\mathrm{ox} 1}$ for large interface trap density.

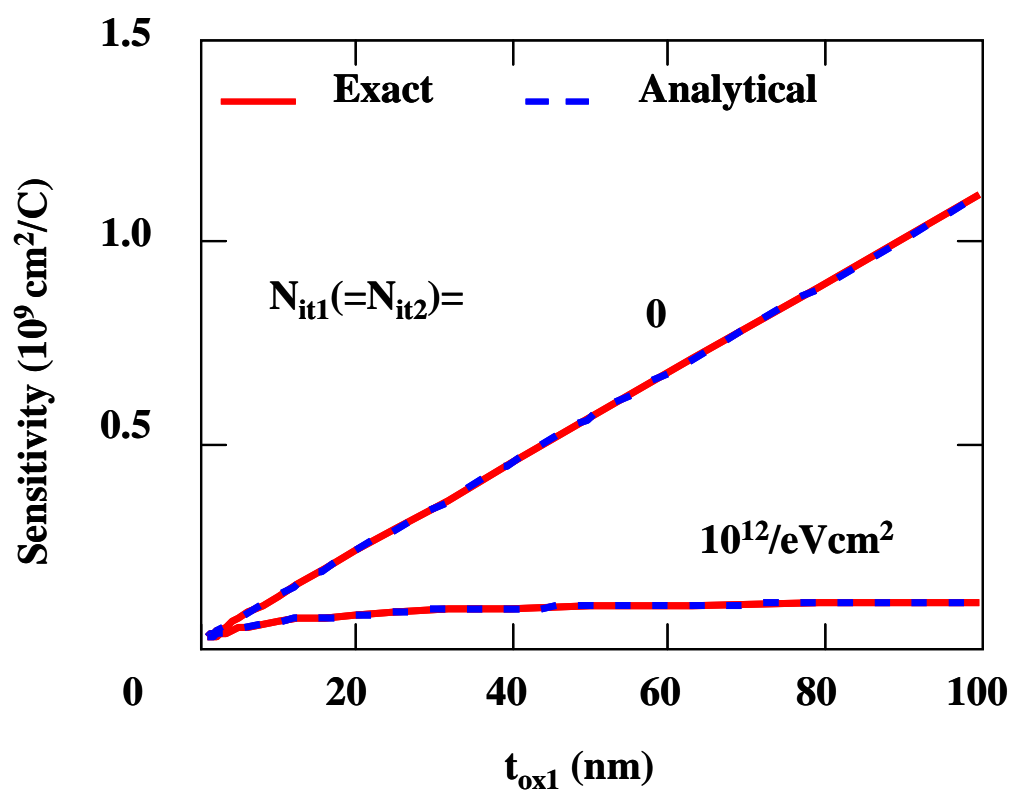

Figure 2. Variation of sensitivity with bottom oxide thickness $t_{o x 1}$. from exact calculations (red solid lines) and analytical expression of Eq. (7) (blue dashed lines) for two interface trap densities with $N_{i t 1}=N_{i t 2}$ in a bottom gate operated FDSOI structure $\left(t_{s i}=10 \mathrm{~nm}, t_{o x 2}=2 \mathrm{~nm}, V_{g 1}=0.01 \mathrm{~V}\right)$.

\subsection{Influence of silicon film thickness}

Typical variations of sensitivity Sens with silicon film thickness $t_{\mathrm{si}}$ are given in Fig. 3 as obtained from exact calculations (red solid lines) and analytical expression of Eq. 7 (blue dashed lines) for two interface trap densities with $\mathrm{N}_{\mathrm{it} 1}=\mathrm{N}_{\mathrm{it} 2}\left(\mathrm{t}_{\mathrm{ox} 1}=10 \mathrm{~nm}, \mathrm{t}_{\mathrm{ox} 2}=2 \mathrm{~nm}, \mathrm{~V}_{\mathrm{g} 1}=0.01 \mathrm{~V}\right)$ in the case of bottom gate operated FDSOI structure. Note also the very good agreement achieved by the analytical equation (7), which well captures the increase of Sens with $t_{s i}$. Similarly, the sensitivity varies almost linearly with $t_{s i}$ for zero interface trap density, whereas it tends to saturate for large interface trap density. 


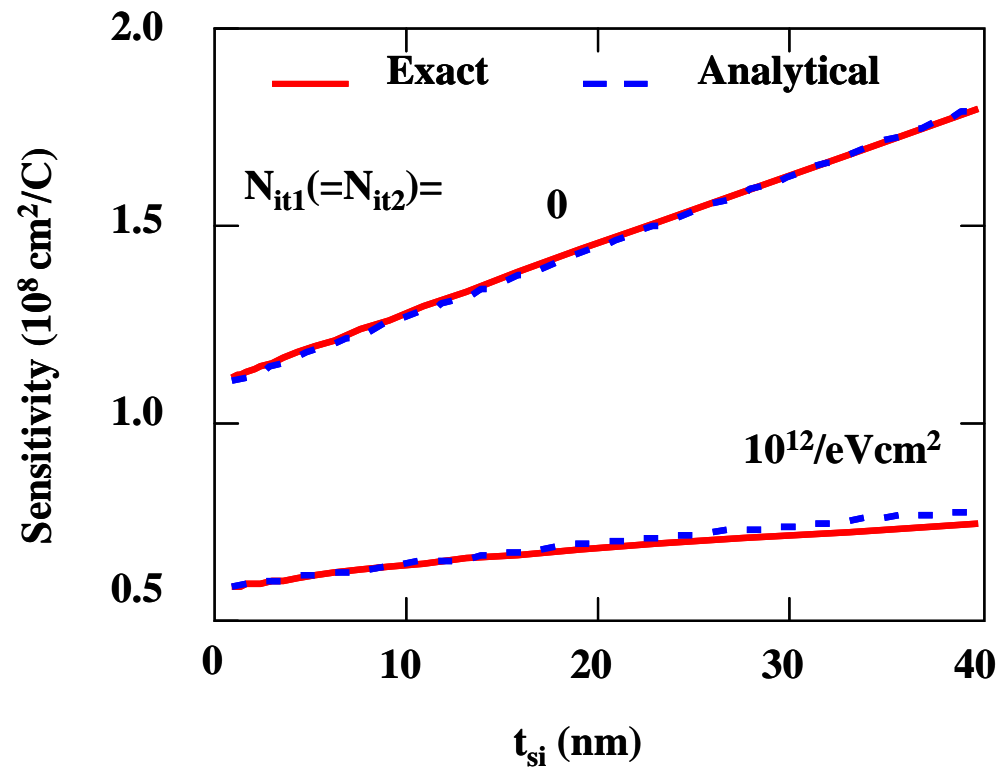

Figure 3. Typical variations of sensitivity with silicon film thickness $t_{\text {si }}$ from exact calculations (red solid lines) and analytical expression of Eq. (7) (blue dashed lines) for two interface trap densities with $N_{i t 1}=N_{i t 2}$ in a bottom gate operated FDSOI structure $\left(t_{o x 1}=10 \mathrm{~nm}, t_{o x 2}=2 \mathrm{~nm}, V_{g 1}=0.01 \mathrm{~V}\right)$.

\subsection{Influence of interface trap density}

Typical variations of sensitivity Sens with interface trap density $\left(\mathrm{N}_{\mathrm{it} 1}=\mathrm{N}_{\mathrm{it} 2}\right)$ are illustrated in Fig. 4 as obtained from exact calculations (red solid lines) and the analytical expression of Eq. (7) (blue dashed lines $)$ in the case of bottom gate operated FDSOI structure $\left(t_{s i}=10 \mathrm{~nm}, t_{\mathrm{ox} 1}=10 \mathrm{~nm}, \mathrm{t}_{\mathrm{ox} 2}=2 \mathrm{~nm}\right.$, $\mathrm{V}_{\mathrm{g} 1}=0.01 \mathrm{~V}$ ). Here also, one should notice the very good description of the sensitivity as a function of the interface trap density provided by Eq. (7) and its strong degradation with increasing the interface trap density.

Finally, typical variations of threshold voltage shift $\Delta \mathrm{V}_{\mathrm{th}}$ for $\mathrm{Q}_{\mathrm{ext}}=10^{11} \mathrm{q} / \mathrm{cm}^{2}$ with silicon film thickness $t_{\mathrm{si}}$ are illustrated in Fig. 5 as obtained from Eq. (9) for two interface trap densities $\mathrm{N}_{\mathrm{it} 1}=\mathrm{N}_{\mathrm{it} 2}$ in a bottom gate operated FDSOI structure $\left(\mathrm{t}_{\mathrm{ox} 1}=10 \mathrm{~nm}, \mathrm{t}_{\mathrm{ox} 2}=2 \mathrm{~nm}\right)$. Note the noticeable reduction of the threshold voltage shift at large silicon film thickness for high trap density due to the increasing electrostatic screening of the top interface trap capacitance $C_{i t 2}$ in Eq. (9).

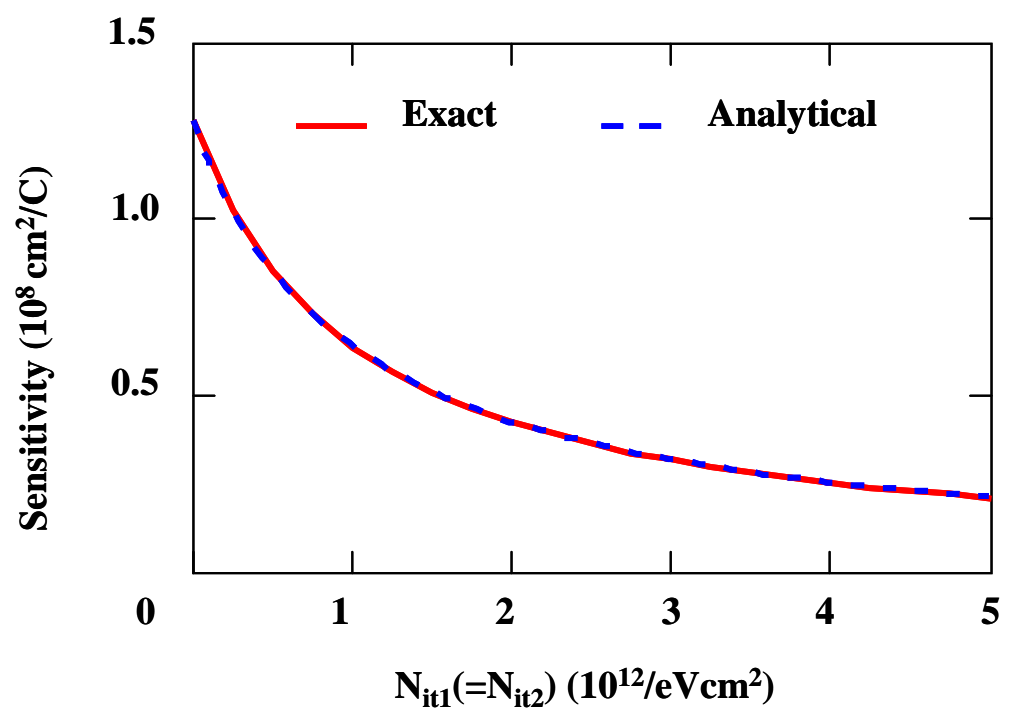

Figure 4. Typical variation of sensitivity Sens with interface trap density $\left(N_{i t 1}=N_{i t 2}\right)$ from exact calculations (red solid lines) and analytical expression of Eq. (7) (blue dashed lines) in a bottom gate operated FDSOI structure

$$
\left(t_{s i}=10 \mathrm{~nm}, t_{o x 1}=10 \mathrm{~nm}, t_{o x 2}=2 \mathrm{~nm}, V_{g 1}=0.01 \mathrm{~V}\right) \text {. }
$$




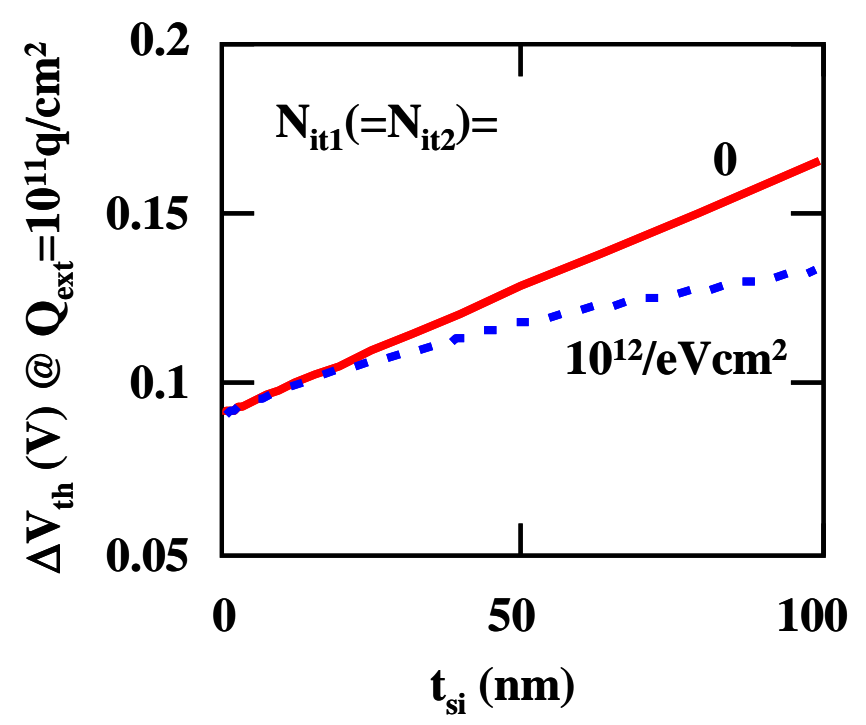

Figure 5. Typical variations of threshold voltage shift $\Delta V_{\text {th }}$ for $Q_{e x t}=10^{11} \mathrm{q} / \mathrm{cm}^{2}$ with silicon film thickness $t_{s i}$ as obtained from Eq. (9) for two interface trap densities $N_{i t 1}=N_{i t 2}$ in a bottom gate operated FDSOI structure

$$
\left(t_{o x 1}=10 \mathrm{~nm}, t_{o \times 2}=2 \mathrm{~nm}\right) \text {. }
$$

\section{Conclusions}

An analytical expression of the free top surface charge sensitivity in FDSOI MOS structure has been established for weak inversion region and validated by TCAD numerical simulation. The influence of various FDSOI stack parameters has been analyzed. The impact of the interface trap density has been particularly emphasized, leading to a strong undesired degradation of sensitivity. This indicates that top surface passivation is a key issue for efficient charge sensing. These expressions of top surface charge sensitivity and associated threshold voltage shift should be very useful for sensor design and electrical characterization purpose.

\section{Acknowledgements}

This work has been partially supported by the H2020 European project Nanonets2sense under $\mathrm{GAn}^{\circ} 688329$.

\section{References}

[BER 03] Bergveld P., Thirty years of ISFETOLOGY: what happened in the past 30 years and what may happen in the next 30 years, Sens Actuators B: Chem, 88, 1 (2003)

[STE 07] Stern E, et al., Label-free immunodetection with CMOS-compatible semiconducting Nanowires, Nature, 445, 519 (2007).

[IBA 05] Iba S, et al. Control of threshold voltage of organic field-effect transistors with double-gate structures, Appl. Phys. Lett., 87, 023509 (2005).

[PAR 14] Park J-K, et al, SOI dual-gate ISFET with variable oxide capacitance and channel thickness, Solid-State Electron, 97, 2 (2014).

[MON 16] Monfray S, Skotnicki T., UTBB FDSOI: evolution and opportunities, Solid-State Electron 125, 63 (2016).

[RAH 17] L. Rahhal, et al, High sensitivity pH sensing on the BEOL of industrial FDSOI transistors, Solid-State Electron, 134, 22 (2017).

[GHI 18] G. Ghibaudo and G. Pananakakis, Analytical expressions for subthreshold swing in FDSOI MOS structures, Solid State Electronics, 149, 57 (2018).

[WOU 90] D. J. Wouters, J. P. Colinge and H. Maes, Subthreshold Slope in Thin-Film SO1 MOSFET's, IEEE Transactions on Electron Devices, 37, 2022, 1990.

[BAL 90] F. Balestra, et al, Analytical Models of Subthreshold Swing and Threshold Voltage for Thin- and Ultra-ThinFilm SOI MOSFET's, IEEE Transactions on Electron Devices, vol. 37, 2303, 1990. 
[MAZ 91] B. Mazhari, et al, Properties of Ultra-Thin Wafer-Bonded Silicon-on-Insulator MOSFET's, IEEE Transactions on Electron Devices, 38, 1289, 1991. 